\title{
OPEN Machine learning algorithms as new screening approach for patients with endometriosis
}

\author{
Sofiane Bendifallah ${ }^{1,2,8 凶}$, Anne Puchar ${ }^{1,2}$, Stéphane Suisse ${ }^{3}$, Léa Delbos ${ }^{4,5}$, \\ Mathieu Poilblanc ${ }^{6,7}$, Philippe Descamps ${ }^{4,5}$, Francois Golfier ${ }^{6,7}$, Cyril Touboul ${ }^{1,2}$, \\ Yohann Dabi ${ }^{1,2}$ \& Emile Daraï ${ }^{1,2}$
}

Endometriosis-a systemic and chronic condition occurring in women of childbearing age-is a highly enigmatic disease with unresolved questions. While multiple biomarkers, genomic analysis, questionnaires, and imaging techniques have been advocated as screening and triage tests for endometriosis to replace diagnostic laparoscopy, none have been implemented routinely in clinical practice. We investigated the use of machine learning algorithms (MLA) in the diagnosis and screening of endometriosis based on 16 key clinical and patient-based symptom features. The sensitivity, specificity, F1-score and AUCs of the MLA to diagnose endometriosis in the training and validation sets varied from 0.82 to $1,0-0.8,0-0.88,0.5-0.89$, and from 0.91 to $0.95,0.66-0.92,0.77-0.92$, respectively. Our data suggest that MLA could be a promising screening test for general practitioners, gynecologists, and other front-line health care providers. Introducing MLA in this setting represents a paradigm change in clinical practice as it could replace diagnostic laparoscopy. Furthermore, this patient-based screening tool empowers patients with endometriosis to self-identify potential symptoms and initiate dialogue with physicians about diagnosis and treatment, and hence contribute to shared decision making.

Endometriosis is defined as an inflammatory condition characterized by endometrial-like tissue outside the uterus $^{1,2}$. The disease is estimated to affect $5-10 \%$ of women in the reproductive period, accounting for about 2.4 million women in France and approximately 190 million women worldwide ${ }^{2,3}$.

Endometriotic lesions can occur at different locations, including the pelvic peritoneum and the ovary, or infiltrate pelvic structures below the peritoneal surface (deep endometriosis) ${ }^{2}$. From a clinical point of view, endometriosis is a highly enigmatic condition with heterogeneous gynecological symptoms a source of systemic effects and impacting the social and psychological wellbeing of a woman, often resulting in decreased work performance $^{4-6}$. In addition, symptoms may overlap with those of other common conditions (e.g., irritable bowel syndrome or interstitial cystitis), making differential diagnosis challenging ${ }^{7}$.

Internationally, work is being undertaken to improve the awareness, diagnosis and treatment of endometriosis ${ }^{8-11}$. A global consortium of investigators in endometriosis recently published its recommendations for research priorities and highlights the challenges of developing a non-invasive screening tool to facilitate and improve diagnosis ${ }^{9,12}$.

In this specific setting, multiple biomarkers ${ }^{13,14}$, genomic analysis ${ }^{15,16}$, questionnaires ${ }^{17-19}$, symptom-based algorithms ${ }^{17,20}$, and imaging techniques ${ }^{21}$ have been advocated as screening and triage tests for endometriosis. However, none of them have been implemented routinely in clinical practice since none are of clinically relevant accuracy -defined by a sensitivity of 0.94 and a specificity of 0.79 - to replace the direct visualization of lesions through laparoscopic surgery ${ }^{13,14,21}$.

\footnotetext{
${ }^{1}$ Department of Obstetrics and Reproductive Medicine, Hôpital Tenon, 4 rue de la Chine, 75020 Paris, France. ${ }^{2}$ Clinical Research Group (GRC) Paris 6: Centre Expert Endométriose (C3E), Sorbonne University (GRC6 C3E SU), Paris, France. ${ }^{3}$ Ziwig Health, 19 rue Reboud, Lyon, France. ${ }^{4}$ Department of Obstetrics and Reproductive Medicine-CHU d'Angers, Angers, France. ${ }^{5}$ Endometriosis Expert Center-Pays de la Loire, La Réunion, France. ${ }^{6}$ Department of Obstetrics and Reproductive Medicine, Lyon South University Hospital, Lyon Civil Hospices, Bron, France. ${ }^{7}$ Endometriosis Expert Center-Steering Center of the EndAURA Network, Paris, France. ${ }^{8}$ Department of Surgical Oncology, Tenon University Hospital, 4 Rue de la Chine, 75020 Paris, France. ${ }^{\square}$ email: sofiane.bendifallah@yahoo.fr
} 
Recent innovation in Artificial Intelligence (AI), Machine Learning (ML), and Deep learning (DL) is emerging as a promising statistical data-driven approach to solve a range of endemic issues, including for endometriosis $^{15,16,20,22,23}$. In addition, wearable sensors ${ }^{20,24,25}$ and smartphones ${ }^{26,27}$ are being explored as a way of connecting medical researchers to patients, and vice versa. With these mobile technologies, patients can provide longitudinal, real-world evidence of their experience. For example, recent software platforms like ResearchKit (http://researchkit.org/) or Ziwig Health (https://ziwig.com/) facilitate the use of mobile technology and AI to recruit patients into studies.

We therefore designed a study (1) to train machine learning algorithms (MLA) to predict the likelihood of endometriosis, and (2) to validate MLA performance on unseen data from the Endo-mi RNA cohort study using the best performing trained models.

\section{Material and method}

Patient-generated data. The training dataset used in this study was pseudonymized data collected between January 2021 to May 2021 from the open health platform, Ziwig Health (https://ziwig.com/). This platform contains 8000 records of patients with symptom suggestive of endometriosis with 500 features about diagnosis, symptoms, imaging, medical treatment, fertility and surgical treatments, and follow-up. To create our training dataset to predict the likelihood of a diagnosis of endometriosis, we filtered the full Ziwig Health dataset to identify patient with diagnosis of endometriosis based on previous treatment for endometriosis or clinical examination confirming deep endometriosis, or sonography/MRI detecting ovarian, peritoneal or deep endometriosis. The control group was composed of patient with at least one symptom suggestive of endometriosis but without previous treatment for endometriosis or clinical examination confirming deep endometriosis, or sonography/MRI detecting ovarian, peritoneal or deep endometriosis. The training dataset included three types of data: numerical, categorical, and text. All the patients gave their consent to the use of their data in accordance with the data protection policy (RGPD), and in compliance with French law and the recommendations of the Commission Nationale de l'Informatique et des Libertés (CNIL). We obtained signed informed consent from all participants in the study. The experimental protocol was approved by le comité de protection des personnes (C.P.P.) Sud-Ouest et Outre-Mer 1 (CPP 1-20-095 ID 10476).

Model training. Generality. Machine Learning, Deep Learning, and ensemble models are trained to developp a diagnostic tool for endometriosis. ML models such as Logistic Regression (LR), Random Forest (RF), Decision Tree (DT), eXtreme Gradient Boosting (XGB), and hard/soft Voting Classifier are considered ensemble learning techniques ${ }^{28-34}$. A flowchart of the training protocols employed in this study is detailed in Fig. 1.

\section{Model overview.}

- Logistic Regression (LR) is a statistical model that uses a logistic function to model a binary dependent variable. Mathematically, a binary logistic model has a dependent variable with two possible values, where the two values are labeled "0" and "1". Outputs with more than two values are modeled by multinomial logistic regression. Logistic Regression is used in various fields, including healthcare and social sciences ${ }^{28}$.

- Decision Tree (DT) is a simple and powerful machine learning model that utilizes any information obtained to find the best classification index of data samples. These classification indexes are the nodes of the DT, which then grow to form the tree structure. The DT model has already been successfully applied to research on public health and health behavior ${ }^{29}$.

- Random Forest (RF) classifier is an ensemble method that trains several DTs in parallel with bootstrapping followed by aggregation, jointly referred as bagging. Bootstrapping indicates that several individual DTs are trained in parallel on various subsets of a training dataset using different subsets of available features. Bootstrapping ensures that each individual DT in the RF is unique, which reduces the overall variance of the RF classifier. For the final decision, RF classifier aggregates the decisions of individual DTs and consequently exhibits good generalization ${ }^{29}$.

- eXtreme Gradient Boosting (XGB) is a gradient boosting algorithm which is an ensemble of weak prediction models, mostly DTs. An individual tree is a simple, often unreliable, model but when multiple trees are grouped together, they can create a robust algorithm. XGB starts by creating a simple tree, which than progresses sequentially and builds upon the weaker learners, with each iteration revising the previous tree until an optimal point is reached, such as the number of trees (estimators) to build the solution ${ }^{34}$.

- Voting Classifier algorithm is a machine learning model that trains on an ensemble of numerous models and predicts an output (class) based on their highest probability of a chosen class as the output. It simply aggregates the findings of each classifier passed into Voting Classifier and predicts the output class based on the highest majority of voting. Voting classifier supports two types of voting: hard voting where the predicted output class is a class with the highest majority of votes; soft voting where the output class is the prediction based on the average of probability given to that class ${ }^{35}$.

- Chi-Square Test: the Chi-square test is one of the most widely used non-parametric tests, often utilized to test the independence between observed and expected frequencies of one or more attributes in a contingency table. In this work, the Chi-square test was used to identify top significant features given the dependent variable $(\mathrm{Y})^{36}$.

The performance of the MLAs was quantified with respect to sensitivity, specificity, F1-score, and discrimination criteria ${ }^{37,38}$ 


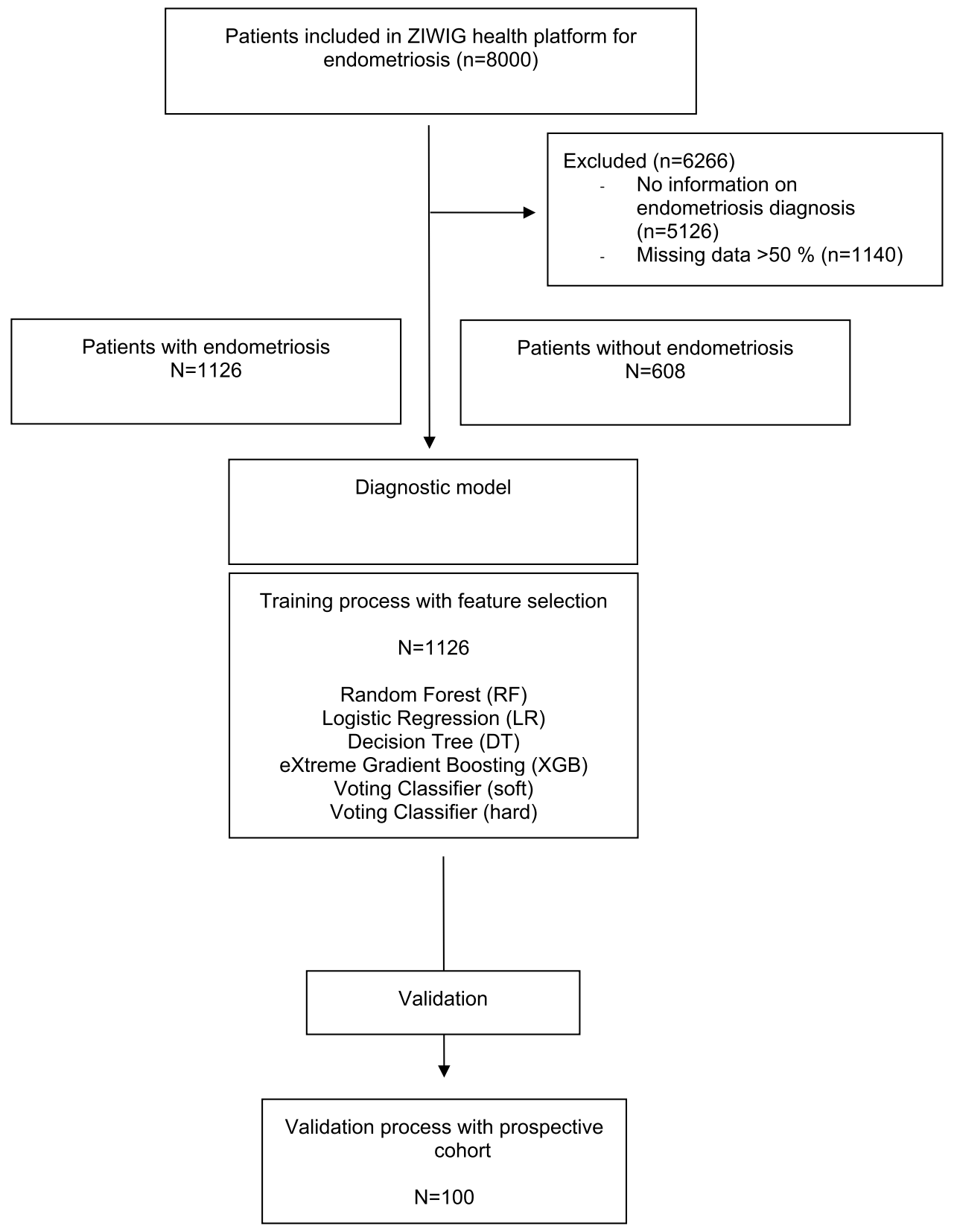

Figure 1. Flow chart of population for model development and validation.

Model validation. The validation dataset was extracted from the prospective ENDOmiARN study (ClinicalTrials.gov Identifier: NCT04728152). The data of the women who participated in the study were aged between 18 and 43 years and had all undergone a laparoscopic procedure, either therapeutic laparoscopy for pain or infertility or diagnostic laparoscopic for chronic pelvic pain. Data collection and the analysis presented in this work were carried out under Research Protocol ( $n^{\circ}$ ID RCB: 2020-A03297-32). For the aim of this study-to predict the likelihood of endometriosis diagnosis-the dataset contained 100 patient records after filtration. The accuracy of the MLAs was quantified with respect to sensitivity, specificity, F1-score, and discrimination criteria ${ }^{37,38}$.

\section{Results}

Epidemiological and surgical characteristics of the dataset. During the study period, 1126 patients and 608 were extracted from Ziwig Health platform (training set) with and without endometriosis to build the diagnostic model. In addition, 100 patients from the prospective cohort (validation set) have been used for the validation. All the patients included in both datasets had a surgical diagnosis of endometriosis. The general and clinical characteristics of the patients in the datasets are summarized in Tables 1 and 2. Significant differences in epidemiological features, symptom history, and medical therapies were found between the datasets. 


\begin{tabular}{|c|c|c|c|}
\hline & $\begin{array}{l}\text { Patient with endometriosis } \\
\mathrm{N}(\%)=1126\end{array}$ & $\begin{array}{l}\text { Patient without endometriosis } \\
\mathrm{N}(\%) \\
\mathrm{N}=608\end{array}$ & $\mathbf{P}<$ value \\
\hline \multicolumn{4}{|l|}{ Demographics characteristics } \\
\hline Age $($ mean \pm SD) & $29 \pm 8$ & $28 \pm 9$ & $<0.001$ \\
\hline BMI (body mass index) (mean \pm SD) & $23.41 \pm 4.88$ & $23.10 \pm 4.56$ & 0.12 \\
\hline \multicolumn{4}{|l|}{ Mother/daughter history of endometriosis } \\
\hline Yes & $21(1.9 \%)$ & $4(0.7 \%)$ & \\
\hline No & $1105(98.1 \%)$ & $604(99.3 \%)$ & 0.056 \\
\hline \multicolumn{4}{|l|}{\begin{tabular}{|l|} 
Endometriosis phenotype \\
\end{tabular}} \\
\hline Dysmenorrhea/VAS of Dysmenorrhea $($ mean \pm SD) & $6 \pm 3.4$ & $5 \pm 3.2$ & $<0.001$ \\
\hline Maximum length of periods (mean \pm SD) & $6 \pm 4$ & $5 \pm 3$ & $<0.001$ \\
\hline \multicolumn{4}{|l|}{ Abdominal pain outside menstruation } \\
\hline Yes & $721(64.1 \%)$ & $179(29.4 \%)$ & $<0.001$ \\
\hline No & $405(35.9 \%)$ & $429(70.6 \%)$ & \\
\hline \multicolumn{4}{|l|}{\begin{tabular}{|l|} 
Pain suggesting sciatica \\
\end{tabular}} \\
\hline Yes & $427(37.9 \%)$ & $61(10.1 \%)$ & \\
\hline No & $699(62.1 \%)$ & $547(89.9 \%)$ & $<0.001$ \\
\hline Pain on sexual intercourse & $3.8 \pm 3.5$ & $2.3 \pm 3.0$ & $<0.001$ \\
\hline \multicolumn{4}{|l|}{ Lower back pain outside menstruation } \\
\hline Yes & $693(61.5 \%)$ & $200(32.9 \%)$ & \\
\hline No & $433(38.5 \%)$ & $408(67.1 \%)$ & $<0.001$ \\
\hline Painful defecation $($ mean \pm SD) & $3.2 \pm 3.3$ & $1.5 \pm 2.4$ & $<0.001$ \\
\hline \multicolumn{4}{|l|}{ Alternating diarrhea/constipation during menstruation } \\
\hline Yes & $718(63.7 \%)$ & $234(38.5 \%)$ & \\
\hline No & $408(36.3 \%)$ & $374(61.5 \%)$ & $<0.001$ \\
\hline Urinary pain during menstruation $($ mean \pm SD) & $1.4 \pm 2.5$ & $0.5 \pm 1.4$ & $<0.001$ \\
\hline \multicolumn{4}{|l|}{ Blood in the stools during menstruation } \\
\hline Yes & $179(15.9 \%)$ & $45(7.4 \%)$ & $<0.001$ \\
\hline No & $947(84.1 \%)$ & $563(92.6 \%)$ & \\
\hline \multicolumn{4}{|l|}{ Blood in urine during menstruation } \\
\hline Yes & $150(13.3 \%)$ & $61(10.1 \%)$ & \\
\hline No & $976(86.7 \%)$ & $547(89.9 \%)$ & 0.046 \\
\hline \multicolumn{4}{|l|}{ Quality of life } \\
\hline Absenteeism duration in the last 6 months $($ mean \pm SD) & $7 \pm 22$ & $3 \pm 12$ & $<0.001$ \\
\hline $\begin{array}{l}\text { Number of non-hormonal pain treatments used } \\
(m e a n \pm S D)\end{array}$ & $1 \pm 1$ & $0 \pm 1$ & $<0.001$ \\
\hline
\end{tabular}

Table 1. Demographic characteristics of the training dataset for patient with and without endometriosis.

For the validation cohort, among those 100 women $87 \%(n=87)$ were diagnosed with endometriosis and $13 \%$ $(n=13)$ without (controls). In both groups, the patients had pain symptoms suggestive of endometriosis. For the endometriosis patients, 51\% (44/87) had rASRM stage I-II, and 49\% (43/87) had stage III-IV. For all patients an MRI has been performed since this information was an inclusion criterion (https://clinicaltrials.gov/ct2/show/ NCT04728152). Concerning the phenotype, among the 87 patients with endometriosis, we reported that 3\% $(n=3 / 87), 6 \%(n=5 / 87), 47 \%(n=41 / 87), 44 \%(n=38 / 87)$ had superficial endometriosis, endometrioma alone, deep infiltrating endometriosis alone, and both deep infiltrating endometriosis + endometrioma.

Selection of significant features in the training set. Pre-processing of dataset. The raw dataset contained 100 features some of which did not significantly affect the prediction of endometriosis occurrence. After taking suggestions from experts in endometriosis (SB, FG, PD, and ED), we selected a total of 16 essential clinical and symptom-based features related to history, demographics characteristics, endometriosis phenotype and treatment (Table 3) free available on the open health platform Ziwig. This approach gives a comprehensive analysis of results where models have been trained and validated on data. A flowchart of the training protocols employed in the study is detailed in Fig. 1.

The top 16 features were used to train the ML model with RF, LR, DT, XGB, Voting Classifier (soft), and Voting Classifier (hard) algorithms (Table 4). A correlation matrix was constructed to reveal the importance of each of the features on the model developed (Figs. 2 and 3). Here we calculated the correlation coefficient between numerical and nominal columns as the Coefficient and the Pearson's chi-square value ${ }^{39}$. 


\begin{tabular}{|c|c|c|c|}
\hline & $\begin{array}{l}\text { Training set } \\
N(\%)=1126\end{array}$ & \begin{tabular}{|l|} 
Validation set \\
$\mathrm{N}(\%)$ \\
$\mathrm{N}=100$
\end{tabular} & $\mathbf{P}<$ value \\
\hline \multicolumn{4}{|l|}{ Demographics characteristics } \\
\hline Age $($ mean $\pm S D)$ & $29 \pm 8$ & $31 \pm 5$ & $<0.001$ \\
\hline BMI (body mass index) (mean \pm SD) & $23.41 \pm 4.88$ & $24.3 \pm 4.82$ & $<0.001$ \\
\hline \multicolumn{4}{|l|}{ Mother/daughter history of endometriosis } \\
\hline Yes & $21(1.9 \%)$ & $8(8 \%)$ & \\
\hline No & $1105(98.1 \%)$ & $92(92 \%)$ & 0.001 \\
\hline \multicolumn{4}{|l|}{ Endometriosis phenotype } \\
\hline Dysmenorrhea/VAS of dysmenorrhea $($ mean $\pm S D)$ & $6 \pm 3.4$ & $7.3 \pm 3$ & $<0.001$ \\
\hline Maximum length of periods (mean \pm SD) & $6 \pm 4$ & $8 \pm 4$ & $<0.001$ \\
\hline \multicolumn{4}{|l|}{ Abdominal pain outside menstruation } \\
\hline Yes & $721(64.1 \%)$ & $67(67 \%)$ & \\
\hline No & $405(35.9 \%)$ & $33(33 \%)$ & 0.5527 \\
\hline \multicolumn{4}{|l|}{ Pain suggesting sciatica } \\
\hline Yes & $427(37.9 \%)$ & $53(53 \%)$ & 0.003 \\
\hline No & $699(62.1 \%)$ & $47(47 \%)$ & \\
\hline Pain on sexual intercourse & $3.8 \pm 3.5$ & $5.1 \pm 3.5$ & $<0.001$ \\
\hline \multicolumn{4}{|l|}{ Lower back pain outside menstruation } \\
\hline Yes & $693(61.5) \%$ & $79(79 \%)$ & 0.00053 \\
\hline No & $433(38.5) \%$ & $21(21 \%)$ & \\
\hline Painful defecation (mean \pm SD) & $3.2 \pm 3.3$ & $4.2 \pm 3.3$ & $<0.001$ \\
\hline \multicolumn{4}{|l|}{ Alternating diarrhea/constipation during menstruation } \\
\hline Yes & $718(63.7 \%)$ & $80(80 \%)$ & \\
\hline No & $408(36.3 \%)$ & $20(20 \%)$ & 0.0010 \\
\hline Urinary pain during menstruation (mean $\pm \mathrm{SD}$ ) & $1.4 \pm 2.5$ & $1.9 \pm 2.9$ & $<0.001$ \\
\hline \multicolumn{4}{|l|}{ Blood in the stools during menstruation } \\
\hline Yes & $179(15.9 \%)$ & $20(20 \%)$ & 0.2862 \\
\hline No & $947(84.1 \%)$ & $80(80 \%)$ & \\
\hline \multicolumn{4}{|l|}{ Blood in urine during menstruation } \\
\hline Yes & $150(13.3 \%)$ & $17(17 \%)$ & 0.3040 \\
\hline No & $976(86.7 \%)$ & $83(83 \%)$ & \\
\hline \multicolumn{4}{|l|}{ Quality of life } \\
\hline Absenteeism duration in the last 6 months $($ mean $\pm S D)$ & $7 \pm 22$ & $23 \pm 31$ & $<0.001$ \\
\hline Number of non-hormonal pain treatments used $($ mean \pm SD) & $1 \pm 1$ & $2 \pm 2$ & $<0.001$ \\
\hline
\end{tabular}

Table 2. Demographic characteristics of the training and validation dataset.

Classification metrics of the training set. The sensitivity, specificity, and F1-score of the 16 features for the MLA to diagnose endometriosis varied from 0.82 to $1,0-0.8,0-0.88$, respectively. Table 4 summarizes the comparison between classification metrics of the different MLAs. Figure 4 summarizes the AUC-ROC curves in the training set.

Classification metrics of validation set. The patient characteristics for the external validation set are summarized in Table 2. Significant differences were found between the patients' phenotype profile compared with the training set. For the 16 most important features selected, the sensitivity, specificity, and F1-score varied from 0.91 to $0.95,0.66-0.92,0.77-0.92$, respectively (Table 4). Figure 5 summarizes the AUC-ROC curves in the validation set.

\section{Discussion}

The present study demonstrates that MLAs based on 16 clinical and symptom-based features enables diagnosis and early prediction of endometriosis onset. The resulting metrics of the model supports the clinical interest of this tool as a screening test for general practitioners (GPs), gynecologists, and other front-line healthcare providers. Patients could also use this tool themselves and it may reduce "diagnostic wandering", and hence diagnostic delay, and result in earlier treatment.

The comparison between the models' metrics supports the clinical value of MLAs as a screening tool to improve the endometriosis patient care pathway with a sensitivity and specificity of $95 \%$ and $80 \%$, respectively. This is in agreement with the Cochrane review of Nisenblat et al. ${ }^{14}$ underlining that the predetermined criteria for a clinically useful non-invasive test to replace diagnostic laparoscopy were a sensitivity and specificity of 0.94 and 0.79 , respectively. Using AI, we confirmed the value of MLA tools with an external validation study on a very 


\begin{tabular}{|l|}
\hline History \\
\hline Mother/daughter history of endometriosis \\
\hline History of surgery for endometriosis \\
\hline Demographics characteristics \\
\hline Age \\
\hline BMI (body mass index) \\
\hline Phenotype \\
\hline Dysmenorrhea/VAS of dysmenorrhea \\
\hline Abdominal pain outside menstruation \\
\hline Pain suggesting of sciatica \\
\hline Pain during sexual intercourse \\
\hline Lower back pain outside menstruation \\
\hline Painful defecation \\
\hline Urinary pain during menstruation \\
\hline Right shoulder pain near or during menstruation \\
\hline Blood in the stools during menstruation \\
\hline Blood in urine during menstruation \\
\hline Quality of life \\
\hline Absenteeism duration in the last 6 months \\
\hline Treatment \\
\hline Number of non-hormonal pain treatments used \\
\hline
\end{tabular}

Table 3. A summary of the 16 dataset features considered in the training approach.

\begin{tabular}{|l|l|l|l|l|l|l|l|l|}
\hline \multirow{2}{*}{ Models } & \multicolumn{2}{l}{ Training set } & \multicolumn{3}{l|}{ Validation set } \\
\cline { 2 - 9 } & Sensitivity & Specificity & F1-score & AUC & Sensitivity & Specificity & F1-score & AUC \\
\hline Random forest (RF) & 0.98 & 0.8 & 0.88 & 0.89 & 0.92 & 0.92 & 0.92 & 0.92 \\
\hline Logistic regression (LR) & 1 & 0 & 0 & 0.5 & 0.95 & 0.81 & 0.87 & 0.88 \\
\hline Decision tree (DT) & 0.82 & 0.8 & 0.81 & 0.82 & 0.91 & 0.66 & 0.77 & 0.78 \\
\hline eXtreme gradient boosting (XGB) & 0.98 & 0.8 & 0.88 & 0.89 & 0.93 & 0.92 & 0.92 & 0.93 \\
\hline Voter classifier soft & 0.98 & 0.6 & 0.74 & 0.75 & 0.93 & 0.88 & 0.9 & 0.90 \\
\hline Voter classifier hard & 0.95 & 0.8 & 0.87 & 0.88 & 0.91 & 0.92 & 0.91 & 0.92 \\
\hline
\end{tabular}

Table 4. Comparison between classification metrics of the different models in the training and validation sets.

different population in terms of endometriosis phenotypes and patient characteristics, suggesting its reproducibility and accuracy. In this specific setting, few data are available on the contribution of AI for the diagnosis and triage of endometriosis. Recently, Kleczyk et al. ${ }^{23}$ validated the role of MLAs for the diagnosis, prediction, and forecasting of endometriosis, based on a medico-economic healthcare database. However, although accurate from a statistical point of view, the clinical utility of this tool is questionable because of (1) the inclusion in the models of key features often associated with other gynecologic disorders such as pelvic inflammatory, sub-mucous myoma or genital infection, (2) the lack of a digital personalized patient-based approach ${ }^{17,40}$, and (3) the lack of external validation to assess its reproducibility. The present MLA tool is a complete patient-based screening questionnaire in accordance with the recent NHS England guidance on patient involvement in their health and care, by which they mean "supporting them to become involved, as much as they want or are able to, in decisions about their care and giving them choice and control" ${ }^{\prime 0}$. It supports the use of self- management approaches that reenforce patients as experts in their own health and provides support to develop understanding and confidence, improved patient experience and adherence to treatment and medication $17,25,27,31,32,40$.

In the last decade, strategies to advance precision medicine have attracted considerable investment in developing new diagnostic methods, treatments, and disease prevention initiatives ${ }^{15,19,26,32,41,42}$. Virtual medical assistants using AI have recently matured and are being used in various health settings ${ }^{15,20,25,30,43}$. In the current study, our MLA screening questionnaire is associated with a sensitivity, specificity, F1-score, and AUC ranging from 0.82 to $1,0-0.8,0-88$, and $0.5-0.89$ in the training and validation sets based on the combination of 16 key common criteria. Interestingly, most of the features included in the MLAs are related to the patient's history, clinical phenotype, and impact on quality of life. Among the MLAs, Soft Voting Classifier, RF and XGB appear the most accurate methods with a sensitivity and specificity ranging between 95 and $98 \%$ and $80 \%$, respectively. Similarly, Yeung et al. developed a predictive model for early endometriosis stages based on a preoperative questionnaire. The model was able to differentiate women with endometriosis from those without (AUC $=0.822$, $\mathrm{P}<0.001$; sensitivity $=80.5 \%$; and specificity $=57.7 \%)$; however, the specificity is low and it cannot be used as 


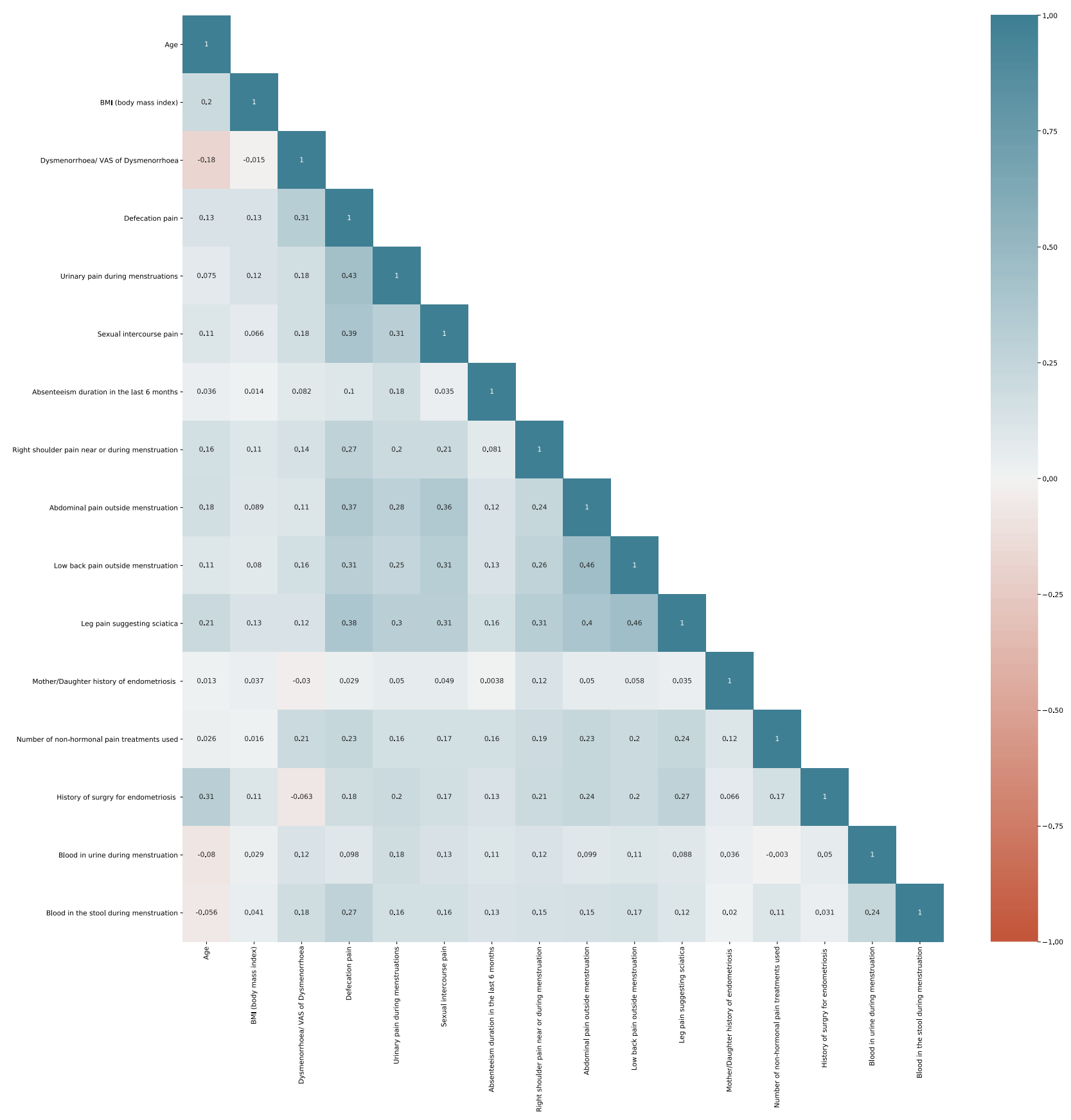

Figure 2. Correlation matrix of 16 features for the training set.

a simple self-completed measure given its complex scoring ${ }^{44}$. In this setting, the scoping review from Surrey et al. ${ }^{17}$ concerning symptom-based screening tools for endometriosis highlighted that only one study evaluated a questionnaire that was solely patient-completed, and that most of the others reported hybrid measures consisting of patient-completed, clinician-completed, imaging, and/or laboratory-based assessments to predict diagnosis.

The strength of the present study is the use of web-based diagnostic tools and symptom checkers that may increase patient health literacy and promote proactive health-seeking behavior. Our diagnostic tool is easily accessible and free for both patients and healthcare providers ${ }^{20,24,26,27}$. Previous studies have underlined the medical contribution of a low-cost method of self-management for healthcare providing effective motivation, and may potentially avoid negative experiences associated with interacting with a health professional who may be perceived as patronizing, judgmental or non-supportive ${ }^{45,46}$. This is especially relevant for endometriosis. Digital interventions may be particularly useful in supporting disadvantaged populations, and particularly adolescents, because user experience less stigmatizing than conventional strategies ${ }^{47}$. Finally, with mobile technologies, patients can provide longitudinal, real-world evidence of their experience. This is of particular relevance for patients seeking to confirm a diagnosis of endometriosis. In a large cohort study, Ballweg et al. ${ }^{48}$ reported that, among patients with symptoms suggestive of endometriosis, $61 \%$ of the healthcare professionals said there was 


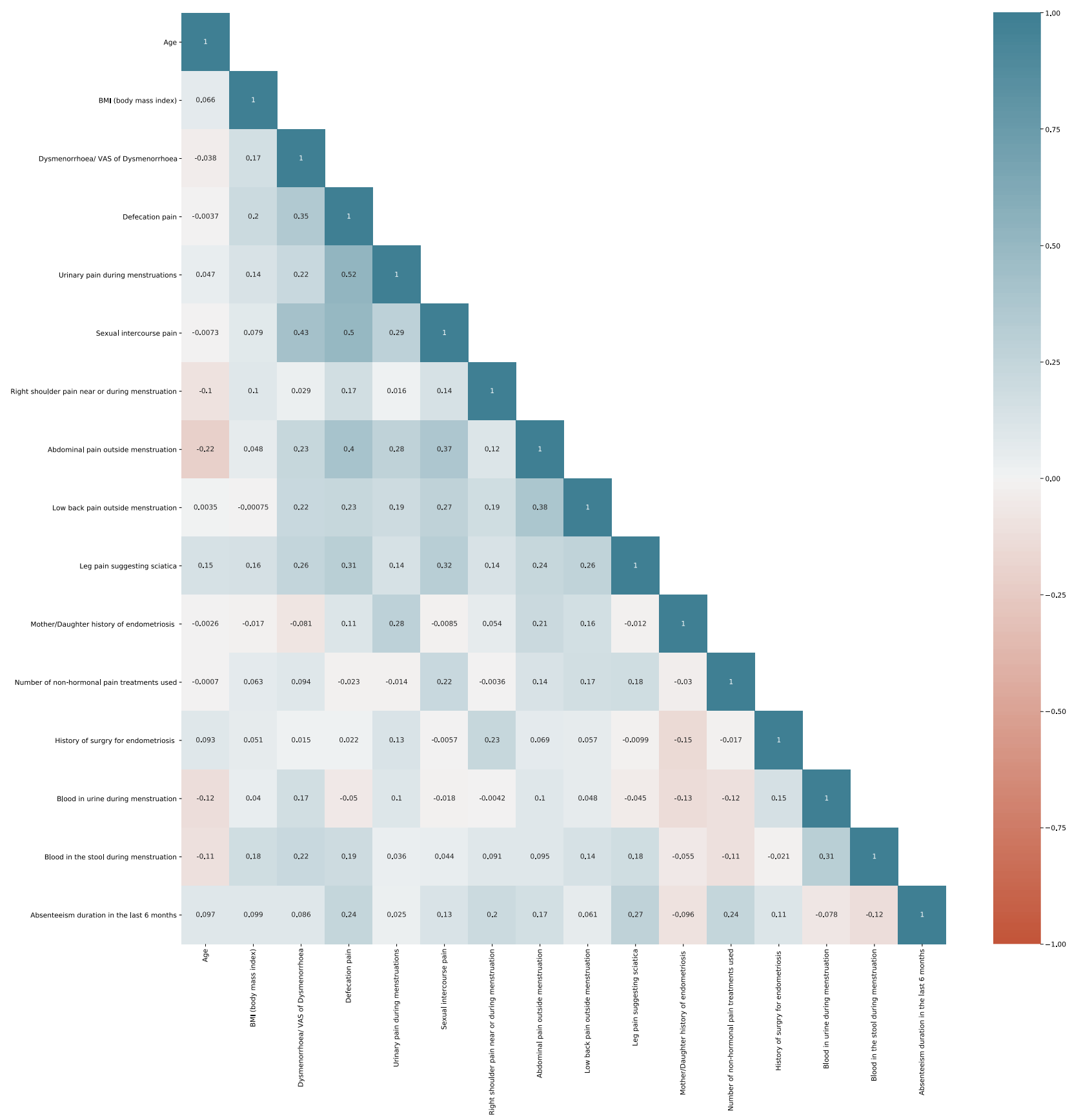

Figure 3. Correlation matrix of the 16 features for the validation set.

"nothing wrong" contributing to a delay in diagnosis. This was confirmed by Greene et al. ${ }^{49}$ who showed that time from onset of symptoms to seeking medical attention and time from seeking medical attention to diagnosis were 4.6 years and 4.7 years, respectively, irrespective of the healthcare provider involved. Hence, the contribution of AI could be crucial as it offers objective data which will improve awareness of endometriosis among healthcare professionals with direct consequences on diagnostic and therapeutic management and the possible referral of patients to expert centers.

In a review of the literature on endometriosis, Zondervan et al. ${ }^{2}$ underlined the low contribution of specific questionnaires as a triage test to diagnose endometriosis. Moreover, clinical examination as well as transvaginal sonography (TVUS) are not always acceptable particularly for adolescents and virgin patients. Bazot et al. ${ }^{50}$ demonstrated that diagnosis of deep endometriosis or endometriomas is easy using TVUS or MRI. However, the meta-analysis of Nisenblat et al. ${ }^{21}$ demonstrated that although diagnosis by TVUS or MRI was accurate for rectal endometriosis and pouch of Douglas obliteration, fulfilling the criteria for SpIN triage tests, imaging techniques were less accurate for other lesions such as utero-sacral ligament endometriosis which is the most frequent location of deep endometriosis. Moreover, imaging techniques have a low accuracy for detecting peritoneal endometriosis which represents the earlier stage of the disease ${ }^{21}$. Conversely, our laparoscopic data 


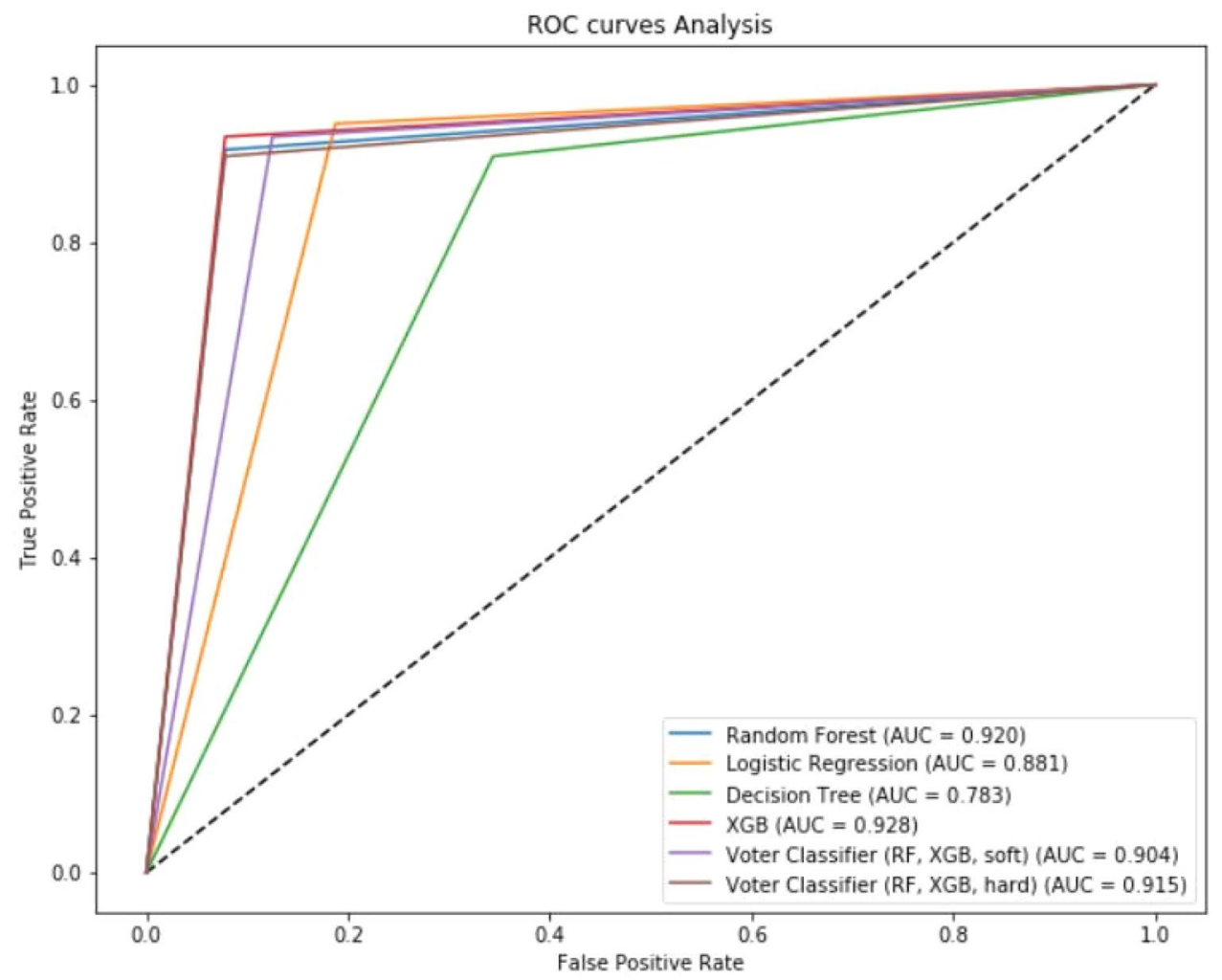

Figure 4. ROC curve analysis of models in training set.

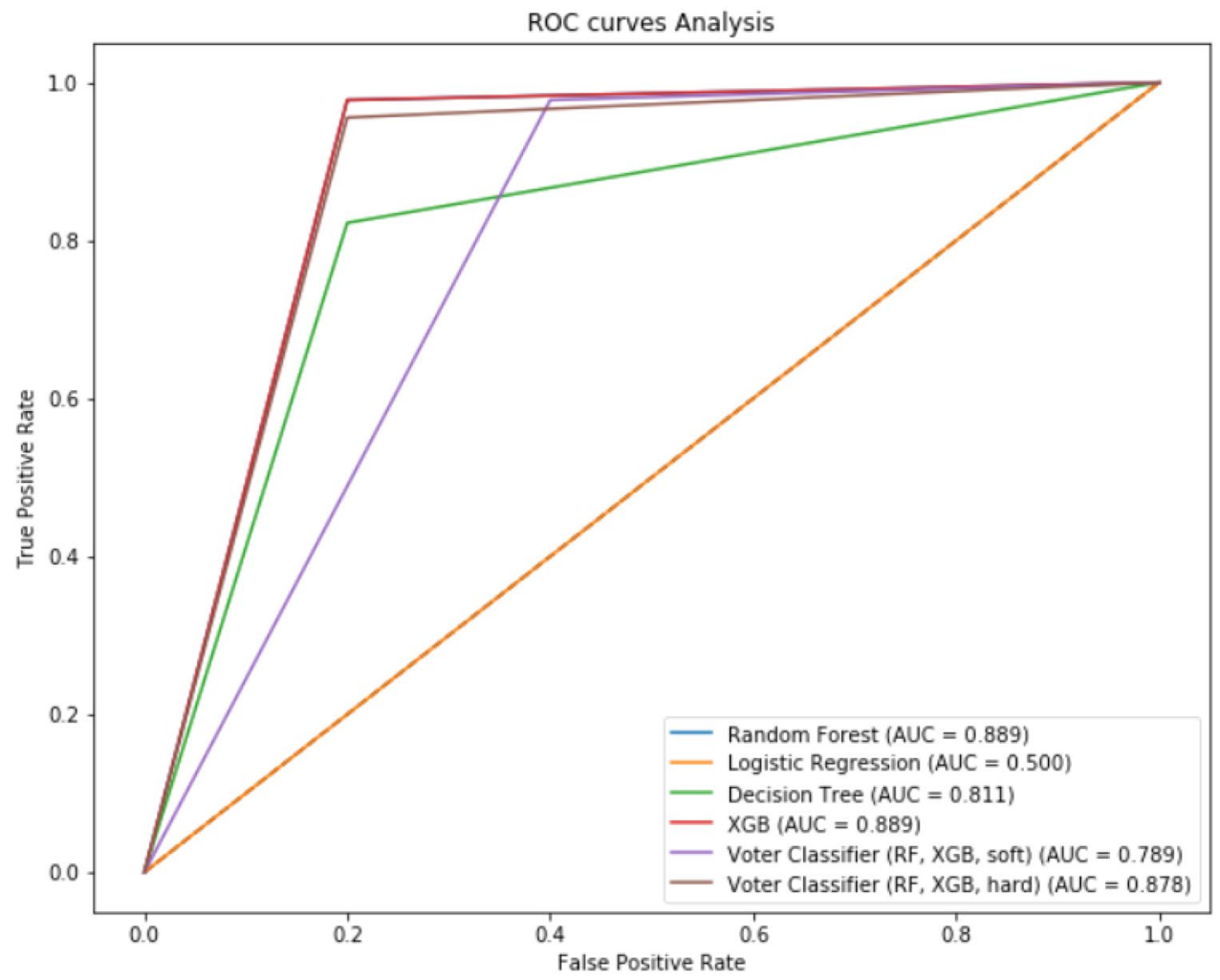

Figure 5. ROC curve analysis of different models in validation set. 
demonstrated that AI alone offers a high accuracy for diagnosing endometriosis even in patients with early disease stage which raises the question of the relevance of diagnostic laparoscopy. Although specialized centers with multidisciplinary teams will surely remain part of the care pathway, particularly for referral from GPs, AI could resolve screening, triaging and assessment issues and help patients navigate the healthcare system which is currently a major concern.

Despite the high accuracy of AI for diagnosing endometriosis, some limitations of the present study deserve to be underlined. First, our population was based on self-questionnaire available on the platform including a large number of items not always fulfilled by the patients with a number of patient with $>50 \%$ at 1140 on 8000 . Moreover, the patient was asked whether there are or not endometriosis with a potential bias in the control group. Indeed, it has been demonstrated that endometriosis could be asymptomatic in up to $20 \%$ of patients ${ }^{21}$. This reinforces the concept of objective test to diagnose endometriosis. Nisenblat et al. underlined that no biomarker of combination of biomarkers can accurately assess the diagnosis of endometriosis ${ }^{21}$. However, a recent study Moustafa et al., suggested the relevance of blood signature of endometriosis based on a limited number of mi RNA, raising the issue to reflect the heterogeneity of endometriosis ${ }^{51}$. This is also underline by Vahnie et al., showing that even using 42 mi RNA no models achieve the value for a SNoUT test ${ }^{14,52}$. Second, the validation set was composed of a relatively small sample size which cannot rule out all potential biases. However, this population was homogeneous and corresponded to patients with suggestive symptoms of endometriosis and having undergone systematic diagnosis of severe endometriosis forms by imaging techniques with surgical confirmation. In this specific setting, Nisenblat et al. demonstrated that imaging techniques for rectal endometriosis had a sensitivity of 0.96 (95\% CI $0.86-0.99)$ and a specificity of 0.98 (95\% CI $0.94-1.00)$, a sensitivity of 0.87 (95\% CI 0.69-0.96) and a specificity of 0.98 (95\% CI 0.95-1.00) for obliterated pouch of Douglas, a sensitivity of 0.82 (95\% CI $0.60-0.95)$ and a specificity of 0.99 (95\% CI 0.97-1.0) for vaginal wall endometriosis, and a sensitivity of 0.88 (95\% CI $0.47-1.0)$ and a specificity of 0.99 (95\% CI $0.96-1.0)$ for rectovaginal septum endometriosis, thus fulfilling the criteria for SpIN triage tests ${ }^{21}$. Moreover, all the patients with early disease stages, who represent a crucial challenge, underwent a diagnostic laparoscopy with systematic biopsy. A second limitation is the absence of patients with discordant features such as symptoms suggestive of endometriosis with negative clinical examination and MRI in the validation set.

In conclusion, our data support the use of MLAs to diagnose endometriosis thereby questioning the relevance of diagnostic laparoscopy and thus constituting a real paradigm change in clinical practice ${ }^{2,13,14}$. Since delays in diagnosis may contribute to undertreatment, continued pain, and prolonged symptom impact which impairs women's quality of life, helping patients to recognize their symptoms is a crucial step toward diagnosis and effective management of endometriosis. Patient-based screening tools empower patients with endometriosis to self-identify potential symptoms and initiate dialogue with physicians about diagnosis and treatment hence contributing to shared decision making.

Received: 4 June 2021; Accepted: 15 December 2021

Published online: 12 January 2022

\section{References}

1. Zondervan, K. T. et al. Endometriosis. Nat. Rev. Dis. Primer 4, 9 (2018).

2. Zondervan, K. T., Becker, C. M. \& Missmer, S. A. Endometriosis. N. Engl. J. Med. 382, 1244-1256 (2020).

3. Shafrir, A. L. et al. Risk for and consequences of endometriosis: A critical epidemiologic review. Best Pract. Res. Clin. Obstet. Gynaecol. 51, 1-15 (2018).

4. Rush, G., Misajon, R., Hunter, J. A., Gardner, J. \& O’Brien, K. S. The relationship between endometriosis-related pelvic pain and symptom frequency, and subjective wellbeing. Health Qual. Life Outcomes 17, 123 (2019).

5. Gallagher, J. S. et al. The impact of endometriosis on quality of life in adolescents. J. Adolesc. Health Off. Publ. Soc. Adolesc. Med. 63, 766-772 (2018).

6. Nnoaham, K. E. et al. Reprint of: Impact of endometriosis on quality of life and work productivity: A multicenter study across ten countries. Fertil. Steril. 112, e137-e152 (2019).

7. Kennedy, S. et al. ESHRE guideline for the diagnosis and treatment of endometriosis. Hum. Reprod. Oxf. Engl. 20, 2698-2704 (2005).

8. Brady, P. C. et al. Research priorities for endometriosis differ among patients, clinicians, and researchers. Am. J. Obstet. Gynecol. 222, 630-632 (2020).

9. Duffy, J. M. N. et al. Top 10 priorities for future infertility research: An international consensus development study. Hum. Reprod. Oxf. Engl. 35, 2715-2724 (2020).

10. van der Zanden, M. et al. Barriers and facilitators to the timely diagnosis of endometriosis in primary care in the Netherlands. Fam. Pract. 37, 131-136 (2020).

11. Hudson, Q. J., Perricos, A., Wenzl, R. \& Yotova, I. Challenges in uncovering non-invasive biomarkers of endometriosis. Exp. Biol. Med. Maywood NJ 245, 437-447 (2020).

12. Horne, A. W., Saunders, P. T. K., Abokhrais, I. M., Hogg, L., Endometriosis Priority Setting Partnership Steering Group (Appendix). Top ten endometriosis research priorities in the UK and Ireland. Lancet Lond. Engl. 389, 2191-2192 (2017).

13. Nisenblat, V. et al. Combination of the non-invasive tests for the diagnosis of endometriosis. Cochrane Database Syst. Rev. https:// doi.org/10.1002/14651858.CD012281 (2016).

14. Nisenblat, V. et al. Blood biomarkers for the non-invasive diagnosis of endometriosis. Cochrane Database Syst. Rev. https://doi. org/10.1002/14651858.CD012179 (2016).

15. Akter, S. et al. GenomeForest: An ensemble machine learning classifier for endometriosis. AMIA Jt. Summits Transl. Sci. Proc. AMIA Jt. Summits Transl. Sci. 2020, 33-42 (2020).

16. Akter, S. et al. Machine learning classifiers for endometriosis using transcriptomics and methylomics data. Front. Genet. 10, 766 (2019).

17. Surrey, E. et al. Patient-completed or symptom-based screening tools for endometriosis: A scoping review. Arch. Gynecol. Obstet. 296, 153-165 (2017).

18. Gater, A. et al. Development and content validation of two new patient-reported outcome measures for endometriosis: the Endometriosis Symptom Diary (ESD) and Endometriosis Impact Scale (EIS). J. Patient-Rep. Outcomes 4, 13 (2020). 
19. Verket, N. J., Falk, R. S., Qvigstad, E., Tanbo, T. G. \& Sandvik, L. Development of a prediction model to aid primary care physicians in early identification of women at high risk of developing endometriosis: cross-sectional study. BMJ Open 9, e030346 (2019).

20. Urteaga, I., McKillop, M. \& Elhadad, N. Learning endometriosis phenotypes from patient-generated data. NPJ Digit. Med. 3, 88 (2020).

21. Nisenblat, V., Bossuyt, P. M., Farquhar, C., Johnson, N. \& Hull, M. L. Imaging modalities for the non-invasive diagnosis of endometriosis. Cochrane Database Syst. Rev. https://doi.org/10.1002/14651858.CD009591.pub2 (2016).

22. Goyal, A., Kuchana, M. \& Ayyagari, K. P. R. Machine learning predicts live-birth occurrence before in-vitro fertilization treatment. Sci. Rep. 10, 20925 (2020).

23. Kleczyk, E. J. et al. Predicting Endometriosis Onset Using Machine Learning Algorithms. https://www.researchsquare.com/article/ rs-135736/v1. (2020). https://doi.org/10.21203/rs.3.rs-135736/v1.

24. Hua, A. et al. Accelerometer-based predictive models of fall risk in older women: a pilot study. NPJ Digit. Med. 1, 25 (2018).

25. Gresham, G. et al. Wearable activity monitors to assess performance status and predict clinical outcomes in advanced cancer patients. NPJ Digit. Med. 1, 27 (2018).

26. Egger, H. L. et al. Automatic emotion and attention analysis of young children at home: A ResearchKit autism feasibility study. NPJ Digit. Med. 1, 20 (2018).

27. Torous, J. et al. Characterizing the clinical relevance of digital phenotyping data quality with applications to a cohort with schizophrenia. NPJ Digit. Med. 1, 15 (2018).

28. Dreiseitl, S. \& Ohno-Machado, L. Logistic regression and artificial neural network classification models: A methodology review. J. Biomed. Inform. 35, 352-359 (2002).

29. Nguyen, J.-M. et al. Random forest of perfect trees: Concept, performance, applications, and perspectives. Bioinforma. Oxf. Engl. https://doi.org/10.1093/bioinformatics/btab074 (2021).

30. Crown, W. H. Potential application of machine learning in health outcomes research and some statistical cautions. Value Health J. Int. Soc. Pharmacoecon. Outcomes Res. 18, 137-140 (2015).

31. Ghassemi, M. et al. A review of challenges and opportunities in machine learning for health. AMIA Jt. Summits Transl. Sci. Proc. AMIA Jt. Summits Transl. Sci. 2020, 191-200 (2020).

32. Sanal, M. G., Paul, K., Kumar, S. \& Ganguly, N. K. Artificial intelligence and deep learning: The future of medicine and medical practice. J. Assoc. Physicians India 67, 71-73 (2019).

33. Lecointre, L. et al. Status of surgical management of borderline ovarian tumors in France: Are recommendations being followed? Multicentric French Study by the FRANCOGYN Group. Ann. Surg. Oncol. https://doi.org/10.1245/s10434-021-09852-9 (2021).

34. Geoffron, S. et al. Fertility preservation in women with malignant and borderline ovarian tumors: Experience of the French ESGOcertified center and pregnancy-associated cancer network (CALG). Gynecol. Oncol. https://doi.org/10.1016/j.ygyno.2021.03.030 (2021).

35. Rocher, G. et al. Does time-to-chemotherapy after primary complete macroscopic cytoreductive surgery influence prognosis for patients with epithelial ovarian cancer? A study of the FRANCOGYN Group. J. Clin. Med. 10(5), 1058 (2021).

36. Jouen, T. et al. The impact of the COVID-19 coronavirus pandemic on the surgical management of gynecological cancers: Analysis of the multicenter database of the French SCGP and the FRANCOGYN group. J. Gynecol. Obstet. Hum. Reprod. 50, 102133 (2021).

37. Harrell, F. E. J., Lee, K. L. \& Mark, D. B. Multivariable prognostic models: Issues in developing models, evaluating assumptions and adequacy, and measuring and reducing errors. Stat. Med. 15, 361-387 (1996).

38. Steyerberg, E. W., Eijkemans, M. J., Harrell, F. E. J. \& Habbema, J. D. Prognostic modelling with logistic regression analysis: A comparison of selection and estimation methods in small data sets. Stat. Med. 19, 1059-1079 (2000).

39. Singhal, R. \& Rana, R. Chi-square test and its application in hypothesis testing. J. Pract. Cardiovasc. Sci. 1, 69 (2015).

40. Ng, K. Y. B. et al. Smartphone-based lifestyle coaching modifies behaviours in women with subfertility or recurrent miscarriage: A randomized controlled trial. Reprod. Biomed. Online. https://doi.org/10.1016/j.rbmo.2021.04.003 (2021).

41. Subramanian, M. et al. Precision medicine in the era of artificial intelligence: Implications in chronic disease management. J. Transl. Med. 18, $472(2020)$.

42. Malvezzi, H., Marengo, E. B., Podgaec, S. \& Piccinato, C. A. Endometriosis: Current challenges in modeling a multifactorial disease of unknown etiology. J. Transl. Med. 18, 311 (2020).

43. Khatibi, T., Hanifi, E., Sepehri, M. M. \& Allahqoli, L. Proposing a machine-learning based method to predict stillbirth before and during delivery and ranking the features: Nationwide retrospective cross-sectional study. BMC Pregnancy Childbirth 21, 202 (2021).

44. Yeung, P. The laparoscopic management of endometriosis in patients with pelvic pain. Obstet. Gynecol. Clin. North Am. 41, 371-383 (2014).

45. Donker, T. et al. Smartphones for smarter delivery of mental health programs: A systematic review. J. Med. Internet Res. 15, e247 (2013).

46. Okorodudu, D. E., Bosworth, H. B. \& Corsino, L. Innovative interventions to promote behavioral change in overweight or obese individuals: A review of the literature. Ann. Med. 47, 179-185 (2015).

47. Dennison, L. et al. Does brief telephone support improve engagement with a web-based weight management intervention? Randomized controlled trial. J. Med. Internet Res. 16, e95 (2014).

48. Ballweg, M. L. Impact of endometriosis on women's health: Comparative historical data show that the earlier the onset, the more severe the disease. Best Pract. Res. Clin. Obstet. Gynaecol. 18, 201-218 (2004).

49. Greene, A. D. et al. Endometriosis: Where are we and where are we going?. Reprod. Camb. Engl. 152, R63-78 (2016).

50. Bazot, M. et al. Diagnostic accuracy of physical examination, transvaginal sonography, rectal endoscopic sonography, and magnetic resonance imaging to diagnose deep infiltrating endometriosis. Fertil. Steril. 92, 1825-1833 (2009).

51. Moustafa, S. et al. Accurate diagnosis of endometriosis using serum microRNAs. Am. J. Obstet. Gynecol. 223(557), e1-557.e11 (2020).

52. Vanhie, A. et al. Plasma miRNAs as biomarkers for endometriosis. Hum. Reprod. Oxf. Engl. 34, 1650-1660 (2019).

\section{Author contributions}

S.B., S.S., M.P., P.D., F.G., E.D. conceived and designed the study. S.B., A.P., Y.D., E.D. included patients and performed the surgical procedures. All authors analyzed the data and wrote the manuscript. All authors have read and agreed to the published version of the manuscript.

\section{Competing interests}

The authors declare no competing interests.

\section{Additional information}

Correspondence and requests for materials should be addressed to S.B.

Reprints and permissions information is available at www.nature.com/reprints. 
Publisher's note Springer Nature remains neutral with regard to jurisdictional claims in published maps and institutional affiliations.

(c) (i) Open Access This article is licensed under a Creative Commons Attribution 4.0 International License, which permits use, sharing, adaptation, distribution and reproduction in any medium or format, as long as you give appropriate credit to the original author(s) and the source, provide a link to the Creative Commons licence, and indicate if changes were made. The images or other third party material in this article are included in the article's Creative Commons licence, unless indicated otherwise in a credit line to the material. If material is not included in the article's Creative Commons licence and your intended use is not permitted by statutory regulation or exceeds the permitted use, you will need to obtain permission directly from the copyright holder. To view a copy of this licence, visit http://creativecommons.org/licenses/by/4.0/.

(C) The Author(s) 2022 